\title{
Análise AMMI da produtividade de grãos em linhagens de soja selecionadas para resistência à ferrugem asiática
}

\author{
Gilberto Ken-Iti Yokomizo(1), João Batista Duarte(2), Natal Antonio Vello(3) e Jair Rogério Unfried(4)
}

\begin{abstract}
(1)Embrapa Amapá, Rodovia JK, Km 05, Caixa Postal 10, CEP 68906-970 Macapá, AP. E-mail: gilberto.yokomizo@embrapa.br (2)Universidade Federal de Goiás, Escola de Agronomia, Campus II, Caixa Postal 131, CEP 74001-970 Goiânia, GO. E-mail: jbduarte@uol.com.br (3)Universidade de São Paulo, Escola Superior de Agricultura Luiz de Queiroz, Departamento de Genética, Avenida Pádua Dias, no 11, Agronomia, Caixa Postal 83, CEP 13400-970 Piracicaba, SP. E-mail: natal.vello@usp.br (4)Tropical Melhoramento e Genética Ltda., Rodovia Celso Garcia Cid, Km 87, Parque Industrial, Caixa Postal 387, CEP 86183-600 Cambé, PR. E-mail: jairunfried@tmg.agr.br
\end{abstract}

Resumo - O objetivo deste trabalho foi quantificar os efeitos da interação genótipo $\mathrm{x}$ ambiente (GxE) sobre a produtividade de grãos em progênies de soja pré-selecionadas para resistência à ferrugem asiática (Phakopsora pachyrhizi). Doze ensaios de avaliação de progênies (linhagens $\mathrm{F}_{6}$ e $\mathrm{F}_{7}$ ) foram conduzidos em diferentes ambientes (combinação de locais, anos e tratamentos fungicidas para controle de doenças de final de ciclo, incluindo ou não a ferrugem). A análise "additive main effects and multiplicative interaction" (AMMI) capturou, como padrão da interação GxE, $57 \%$ da variação associada aos resíduos de não aditividade, dos quais $44 \%$ foram retidos no primeiro componente principal de interação e o restante, no segundo. O primeiro componente associou-se a diferenças entre os anos de avaliação, o que denota imprevisibilidade na predição. O segundo componente, no entanto, associou-se ao manejo diferenciado do cultivo, no que se refere ao controle ou não das doenças. Entre os genótipos de ampla adaptabilidade produtiva, as linhagens USP 02-16.045 e USP 10-10 apresentaram desempenho destacado.

Termos para indexação: Glycine max, Phakopsora pachyrhizi, biplot, interação genótipo x ambiente.

\section{AMMI analysis of grain yield in soybean lines selected for resistance to Asian rust}

\begin{abstract}
The objective of this work was to quantify the effects of genotype $\mathrm{x}$ environment (GxE) interaction on grain yield in soybean progenies pre-selected for resistance to Asian soybean rust (Phakopsora pachyrhizi). Twelve trials evaluating progenies (lines $\mathrm{F}_{6}$ and $\mathrm{F}_{7}$ ) were carried out in different environments (combination of locations, years, and fungicide treatments to control late season diseases, including or not rust). Additive main effects and multiplicative interaction (AMMI) analysis captured, as GxE interaction pattern, 57\% of the variance associated with the residues of non-additivity, of which $44 \%$ were retained in the first principal component of interaction, and the remainder in the second. The first component was associated with the differences between the years of evaluation, which denotes unpredictability. The second component, however, was associated with different crop managements, related to the control or not of the diseases. Among genotypes with wide yield adaptability, the USP 02-16.045 and USP 10-10 lineages stood out.
\end{abstract}

Index terms: Glycine max, Phakopsora pachyrhizi, biplot, genotype x environment interaction.

\section{Introdução}

A soja [Glycine $\max (\mathrm{L}$.$) Merrill] é a oleaginosa com$ maior área plantada no mundo. No Brasil, ela é a cultura anual mais importante, com produção aproximada de 82 milhões de toneladas (Companhia Nacional de Abastecimento, 2013). Apesar da disponibilidade de cultivares para cultivo em diferentes latitudes, tipos de solos e condições climáticas, seu potencial produtivo, que é superior a $4.000 \mathrm{~kg} \mathrm{ha}^{-1}$, dificilmente é alcançado, principalmente pela ocorrência de doenças. Entre essas doenças, a ferrugem asiática, causada pelo fungo Phakopsora pachyrhizi Syd. \& P. Syd., que desde seu surgimento no Brasil, em 2001, até a safra 2008/2009, segundo Del Ponte et al. (2009), teve um custo que ultrapassou US\$ 13 bilhões, valor que leva em consideração as perdas diretas das lavouras, as perdas de arrecadação e o custo do controle químico da doença.

$\mathrm{Na}$ introdução de novas cultivares, normalmente busca-se a compreensão de seu comportamento adaptativo a diferentes sistemas de cultivo e manejo, principalmente em relação à capacidade produtiva e a caracteres morfoagronômicos. Para a soja, em 
razão das diferenças edafoclimáticas dos ambientes de cultivo e da contínua liberação de cultivares, são necessários estudos regionalizados para que se possa avaliar melhor o efeito da interação genótipo $\mathrm{x}$ ambiente (GxE), nas diferentes épocas de semeadura. A espécie é bastante sensível à variação do ambiente (Meotti et al., 2012).

A análise "additive main effects and multiplicative interaction model" (AMMI) vem sendo utilizada com sucesso na avaliação dos efeitos da interação GxE. Vários autores preconizam o método como alternativa adequada para interpretação e compreensão dos diferentes padrões dessa interação (Duarte \& Vencovsky, 1999; Chaves, 2001; Lavoranti, 2003; Gauch, 2006; Gauch et al., 2008; Cravero et al., 2010; Ramalho et al., 2012). Além disso, a análise possibilita fácil representação gráfica dos genótipos e dos ambientes em um diagrama de dispersão multivariado (biplot), o que facilita a sumarização dos dados e a interpretação das relações de similaridade e de complementaridade, entre e dentro de genótipos e ambientes. Essa representação simultânea de cada genótipo e ambiente, além de fornecer informações sobre a estabilidade e a adaptabilidade fenotípica, permite o zoneamento agronômico e a escolha de locais-chave para o processo de avaliação e seleção de cultivares. Em soja, esse tipo de análise vem sendo bastante utilizado (Yan, 2001; Asfaw et al., 2009; Meotti et al., 2012; Martins et al., 2012; Amira et al., 2013).

O objetivo deste trabalho foi quantificar os efeitos da interação genótipo $\mathrm{x}$ ambiente $(\mathrm{GxE})$ sobre a produtividade de grãos em progênies de soja préselecionadas para resistência à ferrugem asiática.

\section{Material e Métodos}

Os experimentos foram realizados durante dois anos agrícolas (2004/2005 e 2005/2006), em três locais do Município de Piracicaba, SP $\left(22^{\circ} 42^{\prime} 30^{\prime \prime S}\right.$, 47³9'00"W, a $540 \mathrm{~m}$ de altitude): Esalq, Anhembi e Areão. O local Esalq consiste na sede do programa de melhoramento de soja da Escola Superior de Agricultura Luiz de Queiroz, da Universidade de São Paulo, e tem solo do tipo Nitossolo Vermelho férrico (antiga Terra Roxa estruturada), de textura argilosa e relevo ondulado. O local Anhembi é caracterizado pelo solo Neossolo Flúvico distrófico, de textura médio-arenosa, relevo plano, e está situado a cerca de $60 \mathrm{~km}$ do local sede. Já o Areão apresenta solo do tipo Argissolo Vermelho-Amarelo distrófico, de textura médio-argilosa, relevo ondulado, e encontra-se a aproximadamente $5 \mathrm{~km}$ do local sede.

Foram avaliadas 28 progênies avançadas (linhagens $\mathrm{F}_{6}$ e $\mathrm{F}_{7}$ ), desenvolvidas no programa de melhoramento mencionado (Tabela 1). Estas linhagens foram préselecionadas entre 1.600 linhagens experimentais, avaliadas na localidade Esalq, em 2003/2004, sob ocorrência severa de ferrugem asiática. $\mathrm{Na}$ ocasião, optou-se por não realizar o controle químico da doença, tendo-se aproveitado a inoculação natural para seleção de linhagens resistentes/tolerantes à doença. As linhagens já haviam sido avaliadas quanto à produtividade de grãos, ciclo, valor agronômico, acamamento e altura da planta na maturidade, nos quatro anos agrícolas anteriores.

O conceito de tolerância adotado baseou-se na produtividade relativa dos quatro anos iniciais vs. a produtividade no ano de ocorrência da ferrugem (Tschanz \& Tsai, 1983; Tschanz \& Wang, 1985). Assim, a partir das linhagens em avaliação, foram selecionadas as 28 presentes em todos os ambientes avaliados, com as maiores produtividades e tolerância à ferrugem e diferencial positivo mínimo de $10 \%$ acima da média das melhores testemunhas do conjunto experimental, no ano de ocorrência da doença. Conforme as diferentes genealogias, nenhuma das linhagens apresentava genes principais de resistência à ferrugem, o que permitiu isolar os efeitos da tolerância condicionada por poligenes, que têm efeitos pequenos e cumulativos, característicos de resistência horizontal (Vello et al., 2002; Pierozzi, 2007).

Os ambientes experimentais perfizeram um total de 12 (Tabela 2), compostos pela combinação de três locais e três tratamentos fungicidas $\left(0,5 \mathrm{~L} \mathrm{ha}^{-1}\right.$ de flutriafol; $0,5 \mathrm{~L} \mathrm{ha}^{-1}$ de carbendazim; e controle, sem aplicação), em dois anos agrícolas. Estes fungicidas são utilizados para controle de doenças de final de ciclo (DFC) da soja. O flutriafol também é indicado para o controle da ferrugem asiática, mas não o carbendazin. Dessa forma, foi possível definir os seguintes contrastes sobre a produtividade de grãos: 1, carbendazim vs. controle, para estimar o efeito das DFC, exceto a ferrugem; 2, flutriafol vs. controle, para estimar o efeito das DFC, incluindo a ferrugem; e 3, flutriafol vs. carbendazim, para estimar o efeito isolado da ferrugem.

Pesq. agropec. bras., Brasília, v.48, n.10, p.1376-1384, out. 2013 DOI: 10.1590/S0100-204X2013001000009 
Utilizou-se o delineamento experimental de blocos ao acaso, com duas (em Anhembi, 2004/2005) ou três repetições (nos demais locais). As parcelas foram compostas por quatro fileiras de $5,0 \mathrm{~m}$ de comprimento, espaçadas a $0,5 \mathrm{~m}$; portanto, tinham $10,0 \mathrm{~m}^{2}$, com área útil de $4,0 \mathrm{~m}^{2}$ (dados obtidos nas duas fileiras centrais da parcela, tendo-se eliminado $0,5 \mathrm{~m}$ em cada extremidade). $\mathrm{O}$ caráter avaliado foi produtividade de grãos.

As análises de variância individuais e conjunta, bem como a análise de estabilidade e adaptabilidade, via modelo AMMI, foram realizadas por meio dos procedimentos GLM e IML do aplicativo computacional SAS (SAS Institute, Cary, NC, EUA). Esta análise, descrita detalhadamente em Duarte \& Vencovsky (1999), baseia-se no seguinte modelo:

$$
Y_{i j}=\mu+g_{i}+e_{j}+\sum_{k=1}^{n} \lambda_{k} \gamma_{i k} \alpha_{j k}+\rho_{i j}+\bar{\varepsilon}_{i j}
$$

Tabela 1. Identificação das linhagens de soja avaliadas $\left(\mathrm{F}_{6}\right.$ e $\left.\mathrm{F}_{7}\right)$, com as respectivas genealogias.

\begin{tabular}{|c|c|c|}
\hline Código & Identificação & Cruzamento \\
\hline 1 & Cruzamento $06-20$ & IAC $4 \times$ Conquista \\
\hline 2 & Cruzamento 06-33 & IAC $4 \times$ Conquista \\
\hline 3 & Cruzamento $10-50$ & Conquista x FT Estrela \\
\hline 4 & Cruzamento $10-52$ & Conquista $\mathrm{x}$ FT Estrela \\
\hline 5 & Cruzamento $11-58$ & Conquista $\mathrm{x}$ Forrest \\
\hline 6 & BUSP-16-015 & (IAC Foscarin-31 x Forrest) x (Foster x FT 79-3.408) \\
\hline 7 & USP $02-16.037$ & (Kirby x FT-2) x Hartwig \\
\hline 8 & USP 02-16.044 & (SOC81-76 x Foster) $x($ Foster x FT 79-3.408) \\
\hline 9 & USP 02-16.045 & (Forrest x Primavera) x (Kirby x FT-2) \\
\hline 10 & USP $02-16.051$ & (IAC Foscarin-31 x Forrest) x (Foster x FT 79-3.408) \\
\hline 11 & USP 02-16.056 & (IAC Foscarin-31 x Forrest) x (Foster x FT 79-3.408) \\
\hline 12 & USP 02-16.071 & (IAC Foscarin-31 x Forrest) x (Foster x FT 79-3.408) \\
\hline 13 & USP 02-16.079 & (Foster x FT 79-3.408) x Hartwig \\
\hline 14 & USP 04-18.029 & Hartwig x MT 123247 \\
\hline 15 & USP $04-18.032$ & Hartwig x (Foster x FT 79-3.408) \\
\hline 16 & USP $04-18.067$ & (IAC-Foscarin-31 x Forrest) x MT 123247 \\
\hline 17 & USP 04-18.083 & (Forrest x Primavera) x (Foster x FT 79-3.408) \\
\hline 18 & USP 09-14 & Foster x FT 79-3.408 \\
\hline 19 & USP $10-10$ & Foster x FT 79-3.408 \\
\hline 20 & USP $10-13$ & Foster x FT 79-3.408 \\
\hline 21 & USP $11-08$ & Foster x FT 79-3.408 \\
\hline 22 & USP $11-38$ & Foster x FT 79-3.408 \\
\hline 23 & USP 11-44 & Foster x FT 79-3.408 \\
\hline 24 & USP 97-08.172 & BR-6 x IAC 100 \\
\hline 25 & USP 97-10.046 & (Primavera x SOC 81-79)x(IAC 10 x GO 81-11.046) \\
\hline 26 & USP 97-11.101 & $\begin{array}{l}{[(\text { Primavera x CEPS } 7716) \times(\text { IAC } 10 \times \text { IAC 12) }] \times} \\
{[(\text { IAC } 4 \times \text { Santa Rosa }) \times(\text { GO } 79-1039 \times \text { Tropical })]}\end{array}$ \\
\hline 27 & BR 16 & Testemunha \\
\hline 28 & IAC-100 & Testemunha \\
\hline
\end{tabular}

em que $Y_{\mathrm{ij}}$ é a resposta média das repetições do i-ésimo genótipo $(i=1,2,3, \ldots, g)$ no $j$-ésimo ambiente $(j=1$, $2,3, \ldots$, e); $\mu$ é a média de todos os genótipos em todos os ambientes (média geral); $\mathrm{g}_{\mathrm{i}}$ é o efeito principal do genótipo $\mathrm{i} ; \mathrm{e}_{\mathrm{j}}$ é o efeito principal do ambiente $\mathrm{j} ; \lambda_{\mathrm{k}}, \gamma_{\mathrm{ik}}$ e $\alpha_{j k}$ são os termos da decomposição singular (DVS) da matriz $\mathrm{GE}_{\mathrm{gxe}}=\left\{(\mathrm{ge})_{\mathrm{ij}}\right\}$, que expressam e capturam o "padrão" associado à interação do genótipo i com o ambiente $\mathrm{j}$, sendo $(\mathrm{ge})_{\mathrm{ij}}$ os desvios de aditividade dos dados $\left(\mathrm{Y}_{\mathrm{ij}}\right)$ em relação aos efeitos principais $\mathrm{g}_{\mathrm{i}} \mathrm{e}$ $\mathrm{e}_{\mathrm{j}} ; \rho_{\mathrm{ij}}$ é um ruído adicional a ser eliminado na análise, relativamente ao termo $(\mathrm{ge})_{\mathrm{ij}}$, tomado tradicionalmente como a própria interação; e $\bar{\varepsilon}_{\mathrm{ij}}$ é o erro experimental médio (em nível de médias de repetições) assumido i.i.d. $\sim \mathrm{N}\left(0, \sigma^{2}\right)$.

O modelo de análise AMMI é ajustado em duas etapas sequenciais: na primeira, os efeitos principais, na parte aditiva do modelo (média geral, efeitos de genótipos e ambientes), são ajustados por análise de variância (Anova) e resultam em um resíduo de não aditividade: $(g \hat{e})_{i j}=Y_{i j}-\bar{Y}_{i .}-\bar{Y}_{. j}+\bar{Y}_{\text {. }}$, isto é, nas estimativas de quadrados mínimos ordinários de $(\mathrm{ge})_{\mathrm{ij}}$; na segunda, a interação (parte multiplicativa do modelo) é ajustada mediante DVS ou análise dos componentes principais aplicada à matriz estimada $\mathrm{GE}_{\mathrm{gxe}}=\left\{(\mathrm{gê})_{\mathrm{ij}}\right\}$ e resulta numa porção denominada "padrão" - a interação propriamente dita ou interação AMMI: $\sum_{\mathrm{k}=1}^{\mathrm{n}} \lambda_{\mathrm{k}} \gamma_{\mathrm{ik}} \alpha_{\mathrm{jk}}-\mathrm{e}$ noutra de "ruído" $\left(\rho_{\mathrm{ij}}\right)$, que deverá ser descartada juntamente com o resíduo Anova.

$\mathrm{Na}$ definição do número de eixos principais a serem retidos para explicar e representar graficamente o padrão relacionado à interação GxE, adotaram-se critérios utilizados por Gauch \& Zobel (1988), tendose levado em consideração a proporção da soma de

Tabela 2. Ambientes experimentais usados na avaliação das linhagens.

\begin{tabular}{lccc}
\hline Ambiente & Safra/Ano & Local & Tratamento \\
\hline A & $2004 / 2005$ & Anhembi & Sem fungicida \\
B & $2004 / 2005$ & Anhembi & Carbendazim \\
C & $2004 / 2005$ & Anhembi & Flutriafol \\
D & $2005 / 2006$ & Anhembi & Sem fungicida \\
E & $2005 / 2006$ & Anhembi & Carbendazim \\
F & $2005 / 2006$ & Anhembi & Flutriafol \\
G & $2005 / 2006$ & Esalq & Sem fungicida \\
H & $2005 / 2006$ & Esalq & Carbendazim \\
I & $2005 / 2006$ & Esalq & Flutriafol \\
J & $2005 / 2006$ & Areão & Sem fungicida \\
K & $2005 / 2006$ & Areão & Carbendazim \\
L & $2005 / 2006$ & Areão & Flutriafol \\
\hline
\end{tabular}


quadrados da interação original $\left(\mathrm{SQ}_{\mathrm{gxE}}\right)$ acumulada até o n-ésimo eixo principal: $\sum_{\mathrm{k}=1}^{\mathrm{n}} \lambda_{\mathrm{k}}^{2} / \mathrm{SQ}_{\mathrm{GxE}}$, em que $\mathrm{SQ}_{\mathrm{GxE}}=\sum_{\mathrm{i}}(\mathrm{gê})_{\mathrm{ij}}^{2}$. Dessa forma, o ponto de parada que determină a seleção do modelo na família de modelos AMMI (AMMI0, AMMI1, ..., AMMIn) foi obtido com base na significância dos testes $\mathrm{F}_{\text {Gollob, }}$ para os sucessivos termos de interação, tendo-se, também, reunido os demais termos não retidos no modelo selecionado em porções complementares da $\mathrm{SQ}_{\mathrm{GxE}}$, pelo teste $\mathrm{F}_{\text {Cornelius }}$ (Cornelius et al., 1992; Piepho, 1995).

\section{Resultados e Discussão}

A análise de variância com decomposição AMMI da interação $\mathrm{GxE}$, para produtividade de grãos, revelou diferenças significativas $(p<0,01)$ entre progênies e entre ambientes. Isso indica a possibilidade de se selecionar genótipos superiores e que os ambientes de teste foram consideravelmente discrepantes na expressão do caráter (Tabela 3). Os ambientes dominaram a variação associada aos tratamentos (combinação de genótipos e ambientes) e representaram $82 \%$ de sua soma de quadrados. Os genótipos representaram apenas 8\% dessa variação, indício de uma base genética estreita nas linhagens melhoradas de soja. Por último, a interação GxE original (resíduo de não aditividade), também significativa $(\mathrm{p}<0,05)$, correspondeu aos $10 \%$ restantes.

O primeiro eixo principal da análise AMMI (IPCA1), que contém a maior percentagem do padrão associado à interação $\mathrm{GxE}$, compreendeu $44 \%$ da

Tabela 3. Resumo da análise de variância conjunta com desdobramento dos efeitos da interação genótipos $\mathrm{x}$ ambientes para o caráter produtividade de grãos $\left(\mathrm{kg} \mathrm{ha}^{-1}\right)$, nos anos agrícolas 2004/2005 e 2005/2006.

\begin{tabular}{|c|c|c|}
\hline Fonte de variação & GL & Quadrado médio \\
\hline Genótipos (G) & 27 & $2.294 .884,0 * *$ \\
\hline Ambientes (E) & 11 & $57.186 .637,9 * *$ \\
\hline Interação GxE & 297 & $250.045,3 *$ \\
\hline IPCA1 & 37 & $917.805,6^{* *}$ \\
\hline IPCA2 & 35 & $297.241,0 * *$ \\
\hline IPCA3 & 33 & $230.888,5^{\mathrm{ns}}$ \\
\hline IPCA4 & 31 & $208.475,1^{\mathrm{ns}}$ \\
\hline Resíduo & 690 & $203.821,8$ \\
\hline Total & 1.025 & - \\
\hline Média $\left(\mathrm{kg} \mathrm{ha}^{-1}\right)$ & & 2.330 \\
\hline $\mathrm{CV}(\%)$ & & 19,4 \\
\hline
\end{tabular}

$\mathrm{SQ}_{\mathrm{GxE}}$ (Tabela 4). Esta proporção encontra-se dentro da variação comumente observada, de 26 a 71\%, em pesquisas de mesma natureza com soja (Oliveira et al., 2003; Rocha et al., 2004; Maia et al., 2006; Oliveira et al., 2006). Isso indica que, para produtividade de grãos, nas condições avaliadas, cerca de metade da variação relacionada aos desvios de aditividade dos efeitos principais (genótipos e ambientes) pode ser capturada pelo primeiro componente principal de interação. Além disso, o resultado também indica que um modelo relativamente simples (com poucos termos multiplicativos) pode apresentar boa capacidade preditiva para o comportamento diferencial dos genótipos, nos ambientes avaliados. Este resultado está de acordo com outras pesquisas (Pacheco, 2004; Cravero et al., 2010; Ebdon \& Gauch, 2011).

Ao se considerar a contribuição dos demais eixos principais de interação, observou-se significância estatística $(p<0,01)$ de apenas mais um deles, o IPCA2, com $13 \%$ da variação (Tabela 4). Juntos, os dois primeiros termos explicaram $58 \%$ da $\mathrm{SQ}_{\mathrm{GxE}}$, porção considerada como o "padrão" associado à interação $\mathrm{GxE}$, para o conjunto modelo-dados analisado. Portanto, com essa abordagem, os demais termos (IPCA3 e IPCA4) foram descartados para a descrição da interação GxE, por serem constituídos, principalmente, de ruídos.

O número de eixos principais retidos para explicar e representar graficamente o padrão de interação GxE pode ser variável. Rharrabti et al. (2003), em trigo duro (Triticum durum L.), obtiveram entre dois e quatro eixos, conforme o caráter considerado na análise. Vicente et al. (2004) relataram que, de nove eixos estimados para descrever a interação GxE em soja, quatro foram significativos. No trabalho de Tarakanovas \& Sprainaitis (2005), com trevo branco

Tabela 4. Autovalores $\left(\lambda_{\mathrm{k}}^{2}\right)$ e percentagens, individual (PI) e acumulada (PA), da soma de quadrados total $\left(\mathrm{SQ}_{\mathrm{GxE}}\right)$ associada aos quatro primeiros eixos principais de interação (IPCA's), estimados por análise AMMI para produtividade de grãos em soja.

\begin{tabular}{lccc}
\hline Eixos singulares & \multicolumn{1}{c}{$\lambda_{\mathrm{k}}^{2}$} & PI & PA \\
\hline IPCA1 & $33.958 .808,76$ & 0,44 & 43,76 \\
IPCA2 & $10.403 .433,80$ & 0,13 & 57,17 \\
IPCA3 & $7.619 .321,63$ & 0,10 & 66,98 \\
IPCA4 & $6.462 .729,42$ & 0,08 & 75,31 \\
\hline SQ $_{\text {GxE }}$ & $77.602 .396,62$ & - & - \\
\hline
\end{tabular}

Pesq. agropec. bras., Brasília, v.48, n.10, p.1376-1384, out. 2013 DOI: 10.1590/S0100-204X2013001000009 
(Trifolium repens L.), os três primeiros eixos foram significativos para produtividade de grãos. Campbell \& Jones (2005) obtiveram dois e três eixos significativos, ao avaliarem produção e qualidade de fibra, em algodão (Gossypium hirsutum L.). No entanto, Oliveira et al. (2003), Pacheco (2004) e Maia et al. (2006) optaram por selecionar apenas o primeiro eixo principal, em soja. Neste caso, os eixos explicaram entre 30 e $40 \%$ da $\mathrm{SQ}_{\mathrm{GxE}}$.

Nos estudos em que apenas os dois primeiros eixos (IPCA1 e IPCA2) foram significativos, os resultados da percentagem de explicação de $\mathrm{SQ}_{\mathrm{GxE}}$ variaram de 61 a 88\% (Borges et al., 2000; Yan \& Hunt, 2002; Freire Filho et al., 2003; Campbell \& Jones (2005); Tarakanovas $\&$ Sprainaitis, 2005; Kaya et al., 2006). No presente trabalho, apesar de esta percentagem ter sido menor (57,2\%), a significância apenas dos dois primeiros eixos mostra que eles são suficientes para capturar o "padrão" associado à interação GxE de interesse.

Os genótipos ou ambientes cujos pontos se situam mais próximos à origem do sistema de coordenadas do gráfico biplot são considerados mais estáveis (Duarte \& Vencovsky, 1999; Lavoranti, 2003). Assim, nos gráficos dos modelos AMMI1 (Figura 1) e AMMI2 (Figura 2), observa-se que a maior parte dos genótipos apresentou relativa dispersão, indício de interação com os ambientes (adaptações específicas). No biplot AMMI1 (médias vs. IPCA1), verificou-se que os genótipos mais estáveis, isto é, com menor contribuição para a interação $\mathrm{GxE}$ captada pelo primeiro eixo principal (IPCA1), foram: USP 02-16.045 (9), USP 02-16.056 (11), USP 02-16.071 (12), USP 04-18.029 (14), USP 04-18.032 (15), USP 10-10 (19) e USP 97-08.172 (24). Portanto, esses genótipos devem ser considerados estáveis, haja vista a pequena interação GxE que manifestaram em suas respostas fenotípicas. Entre estes, as linhagens USP 02-16.045 (9) e USP 10-10 (19), que tiveram produtividades médias relativamente elevadas, também podem ser consideradas amplamente adaptadas aos ambientes avaliados.

Entre os genótipos de bom potencial produtivo, destacaram-se também: BUSP-16-015 (6), USP 09-14 (18), USP 10-12 (20), USP 11-08 (21), USP 11-38 (22), USP 11-44 (23) e USP 97-10.046 (25). À exceção deste último, todos os demais tiveram as cultivares norte-americanas Forrest ou Foster como um dos parentais. Essas cultivares são duas fontes de genes de resistência ao nematoide de cisto da soja (NCS) (Heterodera glycines Ichinohe) raça 3 ou 6 (Hiromoto, 1996). Além disso, eles também apresentam aparente

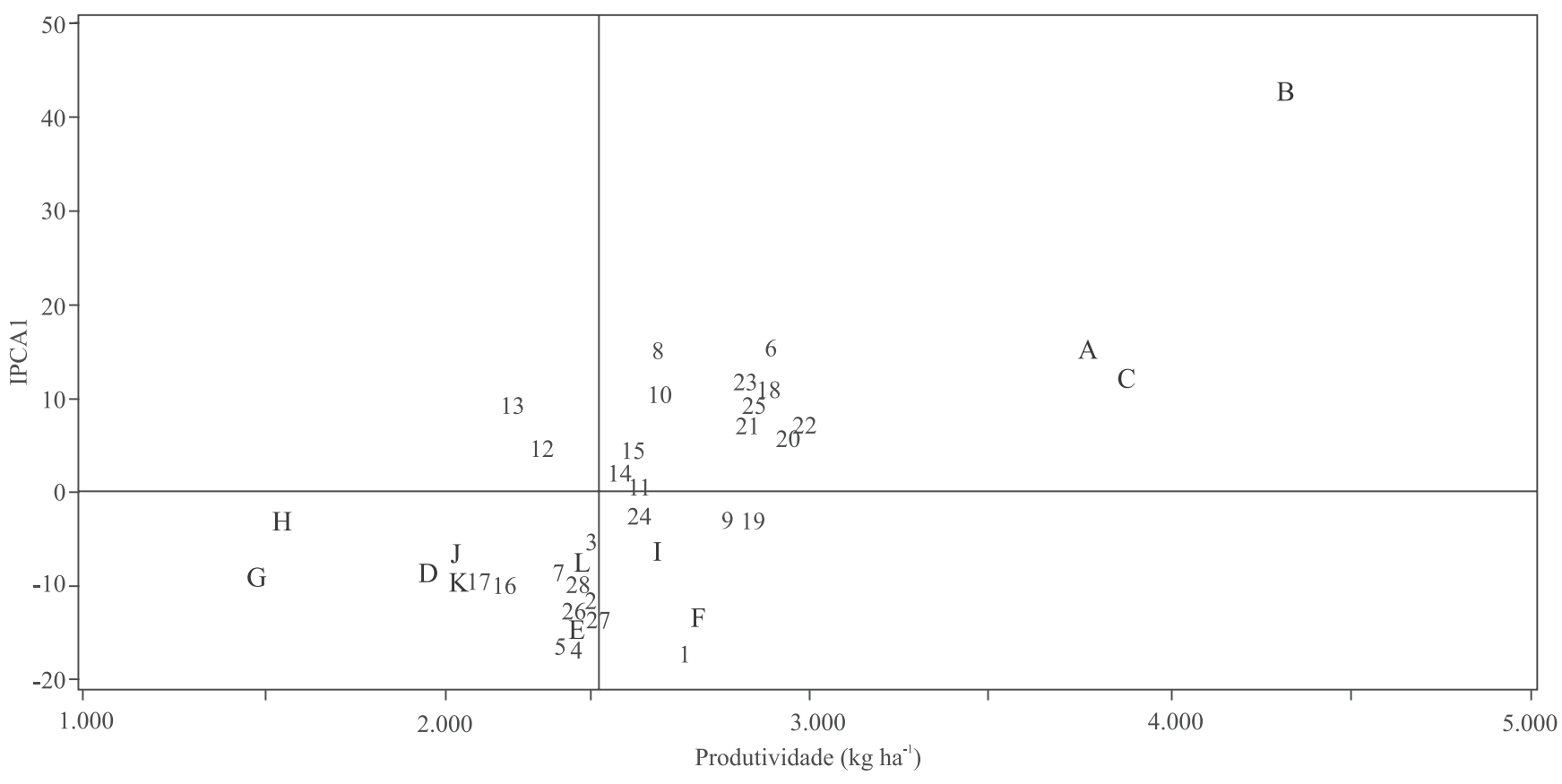

Figura 1. Biplot da análise AMMI1 (produtividade vs. IPCA1) da produtividade de grãos de 28 progênies de soja (códigos de 1 a 28, Tabela 1), avaliadas em doze ambientes (códigos A a L, Tabela 2), nos anos agrícolas 2004/2005 e 2005/2006. 
tendência de adaptabilidade específica aos ambientes A, B e C, principalmente em razão do índice pluvial um pouco maior e mais bem distribuído em 2004/2005 do que em 2005/2006 (Escola Superior de Agricultura Luiz de Queiroz, 2006). Esses dados podem ser constatados pela proximidade das representações gráficas destes genótipos e dos três ambientes, no quadrante superior direito da Figura 1. Genótipos como USP 06-20 (1), USP 10-52 (4) e USP 11-58 (5), de maneira contrária, apresentaram especificidade adaptativa aos demais ambientes avaliados ( $\mathrm{D}$ a $\mathrm{L}$ ), representados na parte inferior da Figura 1.

No biplot AMMI2 (Figura 2), de modo geral, pôdese ratificar o comportamento genotípico observado na análise anterior. Assim, o primeiro eixo principal de interação (IPCA1) captou grande parte do padrão da interação GxE presente no caráter. Entretanto, dada a significância também do segundo eixo (IPCA2), a informação capturada por este pode ser útil para corrigir possíveis distorções na análise ou na interpretação produzida sob uma única dimensão. Portanto, confirmam-se o comportamento estável (baixa contribuição para interação $\mathrm{GxE}$ ) e a ampla adaptabilidade produtiva dos genótipos: USP 10-50 (3), USP 02-16.045 (9), USP 04-18.029 (14), USP
10-10 (19) e USP 97-08.172 (24). Contudo, USP 02-16.056 (11), USP 02-16.071 (12) e USP 04-18.032 (15), aparentemente estáveis na análise sob apenas um só eixo de interação, revelaram agora contribuição para a interação GxE capturada no segundo eixo principal, o que contraria suas estabilidades produtivas. Isso evidencia que o ponto de corte do IPCA1 (43,76\%), em geral, não foi significativo para que se possa inferir sobre a previsibilidade das linhagens quanto à variável produtividade, provavelmente por tratar-se de variável métrica. Essas cultivares, entretanto, mostraram adaptabilidade específica aos ambientes A, C e H.

Um aspecto importante a ser mencionado é que os fatores relacionados à interação GxE explicada pelo primeiro eixo são estatisticamente independentes dos que determinam a interação capturada pelo segundo eixo; dessa forma, não deve haver perda de generalidade em casos que envolvam maior número de eixos selecionados. Assim, esses fatores e seus constructos matemáticos (eixos ou componentes principais) não podem ser desconsiderados na interpretação, caso a magnitude de seus efeitos tenha se revelado significativa. Ademais, a independência entre as variáveis latentes que compõem cada um desses eixos e, por conseguinte, a independência entre

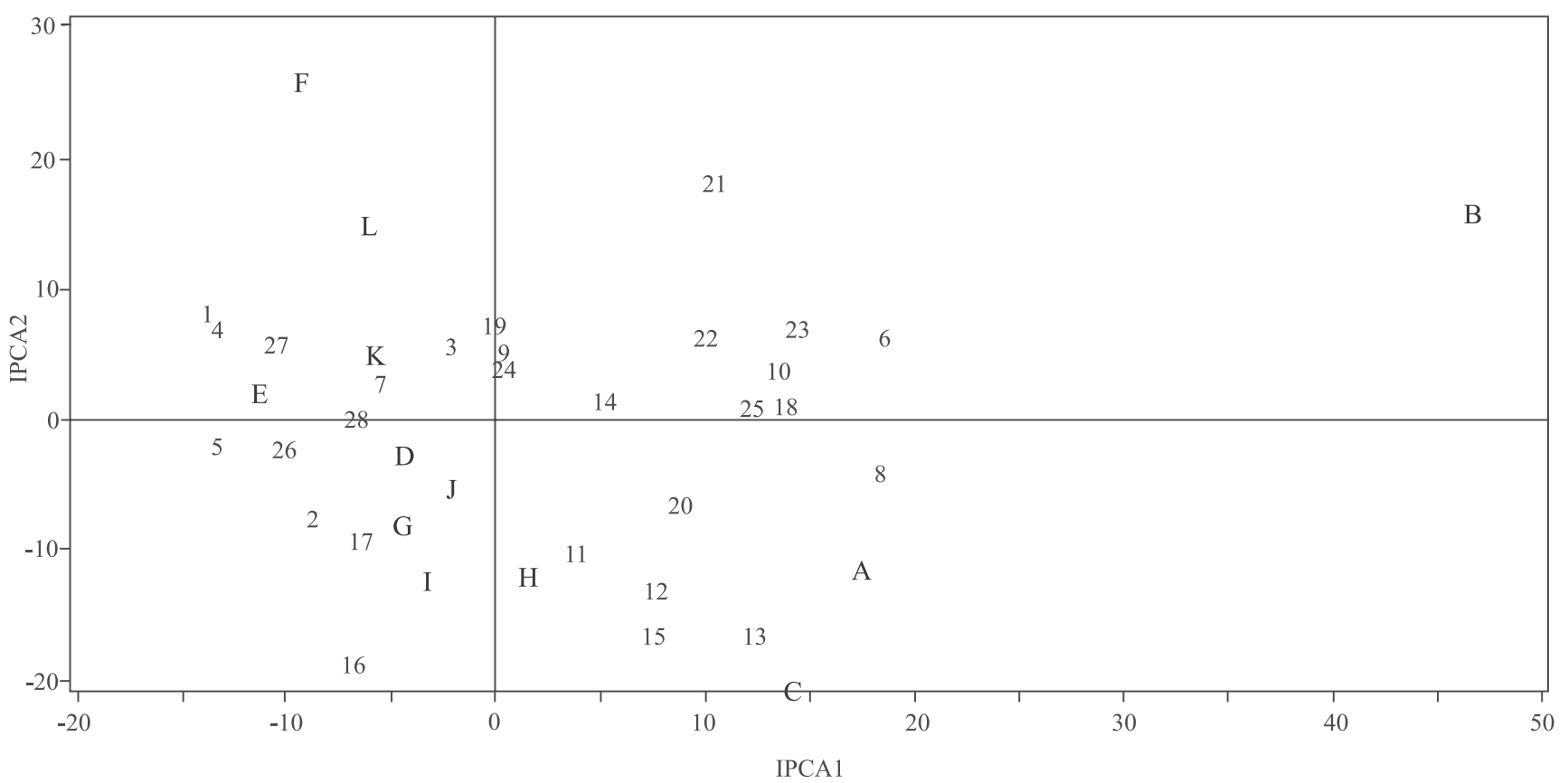

Figura 2. Biplot AMMI2 (IPCA1 vs. IPCA2) da produtividade de grãos de 28 progênies de soja (códigos de 1 a 28 , Tabela 1), avaliadas em doze ambientes (códigos A a L, Tabela 2), nos anos agrícolas 2004/2005 e 2005/2006. 
os fatores ambientais e genéticos a eles subjacentes garantem que, ao se priorizar o manejo de fatores ambientais, identificados segundo a abordagem que fundamenta a análise AMMI, não se lidará com variáveis redundantes.

O refinamento na interpretação da análise, ao se agregar a informação do segundo eixo de interação, permite corrigir ou ratificar o comportamento adaptativo dos genótipos. Por exemplo, USP 10-13 (20) e USP 11-38 (22), que tiveram as maiores produtividades médias e contribuições relevantes para a interação GxE nos dois eixos, agora mostraram adaptabilidades mais específicas; o primeiro, aos ambientes A, C e $\mathrm{H}$, e o segundo, ao ambiente $\mathrm{B}$ (pontos de genótipo e ambientes no mesmo quadrante, Figura 2).

Interações negativas dos genótipos com determinados ambientes também são ratificadas no biplot AMMI2. É possível confirmar, por exemplo, a instabilidade dos genótipos 06-20 (1), 10-52 (4) e 11-58 (5), e suas baixas adaptações produtivas aos ambientes A, B e C. Isso porque tais genótipos tiveram escores de interação de elevada magnitude nos dois eixos (instabilidade) e distribuíram-se em quadrantes distintos a esses ambientes (Figura 2). Os comportamentos coincidentes nos dois gráficos, conforme já reportado, indicam que o primeiro eixo conseguiu capturar a parcela mais importante do padrão da interação GxE para o caráter em estudo; resultado semelhante ao observado por Lavoranti et al. (2002) e Maia et al. (2006).

Os ambientes avaliados individualmente tenderam a contribuir mais para a interação $\mathrm{GxE}$ do que os genótipos. Isso pode ser constatado pela maior dispersão dos pontos ou vetores associados aos ambientes, em relação aos vetores associados aos genótipos, em ambos os biplots (Figuras 1 e 2). Essa tendência pode ser observada reiteradas vezes em outras pesquisas, o que justifica estudos que identifiquem fatores ambientais específicos envolvidos na manifestação desse tipo de interação. Só assim se poderá mitigar os efeitos indesejáveis dos ambientes e explorar seus efeitos positivos de modo mais eficiente (Yan, 2001; Oliveira et al., 2006; Asfaw et al., 2009; Martins et al., 2012; Meotti et al., 2012).

O contraste entre os ambientes A, B e C, de um lado (safra 2004/2005), e os ambientes D, E e F, de outro (safra 2005/2006), quando apenas o local Anhembi estava envolvido, é indicativo de que os fatores relacionados à diferença entre os dois anos agrícolas foram determinantes na interação associada ao primeiro eixo principal (Figuras 1 e 2). Já os contrastes dentro de locais e anos, que envolvem os ambientes A, D e J (sem fungicidas) vs. B, F e L (com aplicação de fungicidas), indicam que a interação $\mathrm{GxE}$ associada ao segundo eixo principal esteve relacionada ao controle das doenças de final de ciclo (ex. contraste A vs. B), sobretudo quando se incluiu o controle da ferrugem (ex. D vs. F e J vs. L). Outro contraste de ambientes que revelou contribuição no segundo eixo de interação foi H e I vs. K e L; ou seja, Esalq vs. Areão, ambos sob aplicação de fungicidas. Ao se considerar a proximidade entre esses locais $(5 \mathrm{~km}) \mathrm{e}$ suas avaliações no mesmo ano agrícola (2005/2006), é provável que essa contribuição seja decorrente de suas diferenças edáficas, mas não propriamente associadas à textura do solo, cujo contraste principal (Anhembi vs. Esalq) não revelou consistência em nenhum dos dois eixos. De qualquer modo, pode-se afirmar que as diferenças edafoclimáticas e de manejo, pela aplicação ou não dos dois fungicidas, foram determinantes para a manifestação das interações GxE na produtividade de grãos.

\section{Conclusões}

1. A variação experimental foi fortemente influenciada pelos ambientes, e a baixa variação genotípica observada indica estreita base genética entre linhagens melhoradas de soja.

2. A análise AMMI foi capaz de capturar $57 \%$ da variação associada aos resíduos de não aditividade, dos quais $44 \%$ estavam retidos no primeiro componente principal de interação, e 13\%, no segundo componente.

3. O primeiro componente da interação $\mathrm{GxE}$ associou-se a diferenças entre os anos de avaliação e, portanto, apresentou caráter imprevisível; já o segundo componente associou-se ao manejo diferenciado entre os ambientes de cultivo, quanto ao controle das doenças de final de ciclo, incluindo a ferrugem asiática.

4. As linhagens USP 02-16.045 e USP 10-10 destacam-se pelas suas produtividades relativamente altas e ampla adaptação produtiva aos ambientes avaliados.

\section{Agradecimentos}

Ao Conselho Nacional de Desenvolvimento Científico e Tecnológico (CNPq), por concessão de bolsa. 


\section{Referências}

AMIRA, J.O.; OJO, D.K.; ARIYO, O.J.; ODUWAYE, O.A.; AYO-VAUGHAN, M.A. Relative discriminating powers of GGE and AMMI models in the selection of tropical soybean genotypes. African Crop Science Journal, v.21, p.67-73, 2013.

ASFAW, A.; ALEMAYEHU, F.; GURUM, F.; ATNAF, M. AMMI and SREG GGE biplot analysis for matching varieties onto soybean production environments in Ethiopia. Scientific Research and Essay, v.4, p.1322-1330, 2009.

BORGES, L.C.; FERREIRA, D.F.; ABREU, A. de F.B.; RAMALHO, M.A.P. Emprego de metodologias de avaliação da estabilidade fenotípica na cultura do feijoeiro (Phaseolus vulgaris L.). Revista Ceres, v.47, p.89-102, 2000.

CAMPBELL, B.T.; JONES, M.A. Assessment of genotype $\mathrm{x}$ environment interactions for yield and fiber quality in cotton performance trials. Euphytica, v.144, p.69-78, 2005. DOI: 10.1007/s10681-005-4336-7.

CHAVES, L.J. Interação de genótipos com ambientes. In: NASS, L.L.; VALOIS, A.C.C.; MELO, I.S.M.; VALADARES, M.C. (Ed.). Recursos genéticos e melhoramento de plantas. Rondonópolis: Fundação MT, 2001. p.673-713.

COMPANHIA NACIONAL DE ABASTECIMENTO. Acompanhamento de safra brasileira: grãos - safra 2012/2013 - sétimo levantamento - abril 2013. Brasília: Conab, 2013. 28p.

CORNELIUS, P.L.; SEYEDSADR, M.; CROSSA, J. Using the shifted multiplicative model to search for "separability" in crop cultivar trials. Theoretical and Applied Genetics, v.84, p.161-172, 1992. DOI: 10.1007/BF00223996.

CRAVERO, V.; MARTIN, E.; ANIDO, F.L.; COINTRY, E. Stability through years in a non-balanced trial of globe artichoke varietal types. Scientia Horticulturae, v.126, p.73-79 2010. DOI: 10.1016/j.scienta.2010.07.012.

DEL PONTE, E.M.; SPOLTI, P.; GODOY, C.V. Ferrugem asiática da soja: panorama e perspectivas para o manejo. Revista Plantio Direto, v.113, p.16-19, 2009.

DUARTE, J.B.; VENCOVSKY, R. Interação genótipos $\mathbf{x}$ ambientes: uma introdução à análise AMMI. Ribeirão Preto: Sociedade Brasileira de Genética, 1999. 60p.

EBDON, J.S.; GAUCH, H.G. Direct validation of AMMI predictions in turfgrass trials. Crop Science, v.51, p.862-869, 2011. DOI: $10.2135 /$ cropsci2010.03.0158.

ESCOLA SUPERIOR DE AGRICULTURA LUIZ DE QUEIROZ. Base de dados da estação meteorológica. 2006. Disponível em: $<$ http://www.lce.esalq.usp.br/automatica/pagina5.html $>$. Acesso em: 12 out. 2013.

FREIRE FILHO, F.R.; RIBEIRO, V.Q.; ROCHA, M. de M.; LOPES, A.C. de A. Adaptabilidade e estabilidade da produtividade de grãos de caupi enramador de tegumento mulato. Pesquisa Agropecuária Brasileira, v.38, p.591-598, 2003. DOI: 10.1590/ S0100-204X2003000500006.
GAUCH, H.G. Statistical analysis of yield trials by AMMI and GGE. Crop Science, v. 46, p.1488-1500, 2006. DOI: 10.2135/ cropsci2005.07-0193.

GAUCH, H.G.; PIEPHO, H.-P.; ANNICCHIARCO, P. Statistical analysis of yield trials by AMMI and GGE: further considerations. Crop Science, v.48, p.866-889, 2008. DOI: 10.2135/ cropsci2007.09.0513.

GAUCH, H.G.; ZOBEL, R.W. Predictive and postdictive success of statistical analysis of yield trials. Theoretical and Applied Genetics, v.76, p.1-10, 1988. DOI: 10.1007/BF00288824.

HIROMOTO, D.M. Seleção de genótipos de soja para performance agronômica e resistência a Heterodera glycines Ichinohe e Diaphorthe phaseolorum f.sp.meridionales Morgan-Jones. 1996. 84p. Tese (Doutorado) - Universidade de São Paulo, Piracicaba.

KAYA,Y.; AKÇURA, M.; TANER, S. GGE-biplot analysis of multi-environment yield trials in bread wheat. Turkish Journal of Agriculture and Forestry, v.30, p.325-337, 2006.

LAVORANTI, O.J. Estabilidade e adaptabilidade fenotípica através da reamostragem "bootstrap” no modelo AMMI. 2003. 166p. Tese (Doutorado) - Universidade de São Paulo, Piracicaba.

LAVORANTI, O.J.; DIAS, C.T. dos S.; VENCOVSKY, R. Estudo da adaptabilidade e estabilidade fenotípica de progênies de Eucalyptus grandis via metodologia AMMI. Boletim de Pesquisa Florestal, v.44, p.107-124, 2002.

MAIA, M.C.C.; VELLO, N.A.; ROCHA, M. de M.; PINHEIRO, J.B.; SILVA JÚNIOR, N.F. da. Adaptabilidade e estabilidade de linhagens experimentais de soja selecionadas para caracteres agronômicos através de método uni-multivariado. Bragantia, v.65, p.215-226, 2006. DOI: 10.1590/S0006-87052006000200004.

MARTINS, J.A.S.; JULIATTI, F.C. Adaptability and stability of soybean advanced lines of semi early cycle for rust resistance. Crop Breeding and Applied Biotechology, v.12, p.43-51, 2012. DOI: 10.1590/S1984-70332012000100006.

MEOTTI, G.V.; BENIN, B.; SILVA, R.R.; BECHE, E.; MUNARO, L.B. Épocas de semeadura e desempenho agronômico de cultivares de soja. Pesquisa Agropecuária Brasileira, v.47, p.14-21, 2012. DOI: 10.1590/S0100-204X2012000100003.

OLIVEIRA, A.B. de; DUARTE, J.B.; CHAVES, L.C.; COUTO, M.A. Environmental and genotypic factors associated with genotype by environment interactions in soybean. Crop Breeding and Applied Biotechnology, v.6, p.79-86, 2006.

OLIVEIRA, A.B. de; DUARTE, J.B.; PINHEIRO, J.B. Emprego da análise AMMI na avaliação da estabilidade produtiva em soja. Pesquisa Agropecuária Brasileira, v.38, p.357-364, 2003. DOI: 10.1590/S0100-204X2003000300004.

PACHECO, R.M. Estratificação de ambientes em cerrados do Brasil Central para fins de seleção e recomendação de cultivares de soja. 2004. 170p. Tese (Doutorado) - Universidade Federal de Goiás, Goiânia.

PIEPHO, H.P. Robustness of statistical test for multiplicative terms in the additive main effects and multiplicative interaction model for cultivar trial. Theoretical and Applied Genetics, v.90, p.438-443, 1995. DOI: 10.1007/BF00221987.

Pesq. agropec. bras., Brasília, v.48, n.10, p.1376-1384, out. 2013 DOI: 10.1590/S0100-204X2013001000009 
PIEROZZI, P.H.B. Controle genético da resistência da soja à ferrugem asiática: avaliações de severidade em campo experimental. 2007. 123p. Dissertação (Mestrado) - Universidade Estadual de Londrina, Londrina.

RHARRABTI, Y.; GARCÍA DEL MORAL, L.F.; VILLGEAS, D.; ROYO, C. Durum wheat quality in Mediterranean environments III. Stability and comparative methods in analysing GxE interaction. Field Crops Research, v.80, p.141-146, 2003. DOI: 10.1016/S0378-4290(02)00178-8.

RAMALHO, M.A.P.; ABREU, A. de F.B.; SANTOS, J.B. dos; NUNES, J.A.R. Aplicações da genética quantitativa no melhoramento de plantas autógamas. Lavras: Ed. da UFLA, 2012. 522p.

ROCHA, M. de M.; VELLO, N.A.; LOPES, Â.C. de A.; MAIA, M.C.C. Yield stability of soybean lines using additive main effects and multiplicative interaction analysis - AMMI. Crop Breeding and Applied Biotechnology, v.4, p.391-398, 2004.

TARAKANOVAS, P.; SPRAINAITIS, A. Main additive effect and multiplicative interaction analysis of white clover genetic resources. Biologija, v.4, p.38-42, 2005.

TSCHANZ, A.T.; TSAI, M.C. Evidence of tolerance to soybean rust in soybeans. Soybean Rust Newsletter, v.6, p.28-31, 1983.
TSCHANZ, A.T.; WANG, T.C. Interrelationship between soybean development, resistance, and Phakopsora pachyrhizi. In: INTERNATIONAL CONGRESS OF THE SOCIETY FOR THE ADVANCED OF BREEDING RESEARCH IN ASIA AND OCEANIA, 5., 1985, Bangkok. Proceedings. Bangkok: Society for the Advanced of Breeding Research in Asia and Oceania, 1985. p.14-20.

VELLO, N.A.; BROGIN, R.L.; ARIAS, C.A.A. Estratégias de melhoramento para o controle da ferrugem da soja. In: CONGRESSO BRASILEIRO DE SOJA, 3.; CONGRESSO BRASILEIRO DE SOJA E MERCOSOJA, 2., 2002. Londrina. Anais. Londrina: Embrapa Soja, 2002. p.188-196. (Embrapa Soja. Documentos, 180).

VICENTE, D.; PINTO, R.J.B.; SCAPIM, C.A. Análise da adaptabilidade e estabilidade de linhagens elite de soja. Acta Scientiarum. Agronomy, v.26, p.301-307, 2004. DOI: 10.4025/ actasciagron.v26i3.1838.

YAN, W. GGE biplot - a Windows application for graphical analysis of multienvironment trial data and other types of two-way data. Agronomy Journal, v.93, p.1111-1118, 2001. DOI: 10.2134/ agronj2001.9351111x.

YAN, W.K.; HUNT, L.A. Biplot analysis of diallel data. Crop Science, v.42, p.21-30, 2002. DOI: 10.2135/cropsci2002.0021.

Recebido em 6 de maio de 2013 e aprovado em 23 de setembro de 2013 